\title{
Retinal Degeneration
}

National Cancer Institute

\section{Source}

National Cancer Institute. Retinal Degeneration. NCI Thesaurus. Code C34979.

Degeneration of the retina. 